Strategien gegen Hypoglykämien

\section{Jahre HyPOS}

— Für viele Menschen mit Diabetes sind Unterzuckerungen ein häufiges Problem. Trotz einer ersten Diabetesschulung werden Hypoglykämien oft schlecht oder erst spät erkannt - eine Gefahr für betroffene Patienten. Seit fünf Jahren kann das strukturierte Schulungs- und Behandlungsprogramm HyPOS im Rahmen der DiseaseManagement-Programme für Menschen mit Typ-2-Diabetes eingesetzt werden. Es vermittelt insulinpflichtigen Patienten Strategien für einen besseren Umgang mit $\mathrm{Hy}$ poglykämien im Alltag. Teilnehmer lernen, diese besser wahrzunehmen, zu vermeiden und zu bewältigen. Entwickelt wurde HyPOS vom Forschungsinstitut der DiabetesAkademie Bad Mergentheim mit Unterstützung der Berlin-Chemie AG.

www.hypos.de

\section{Zubehörsets \\ Abläufe vereinfachen}

— Mit Set2go von Dräger können sich Krankenhäuser jetzt Verbrauchsmaterialien und Zubehörteile für Beatmung und Anästhesie nach eigenen Wünschen in einem Set zusammenstellen lassen. Für die Nachbestellung wird dadurch nur noch eine Sachnummer benötigt. Dadurch verringert sich der Aufwand für Handling und Logistik der Artikel.

Das Bereitstellen, Lagern und Nachbestellen jedes einzelnen Artikels verursacht einen nicht unerheblichen logistischen, aber auch kaufmännischen Aufwand. Set2go vereinfacht diesen Prozess, indem die jeweils benötigten Zubehör- und Verbrauchsmaterialen bereits fertig in einem stabilen Beutel zusammengestellt sind. Dazu ermitteln Dräger-Experten den individuellen Bedarf, so dass eine Klinik immer die für sie maßgeschneiderten Sets erhält und mit nur einer Sachnummer ordern kann. Krankenhäuser können sich Sets aus über 80 unterschiedlichen Einwegmaterialien zusammenstellen lassen.

www.draeger.com

Mangelernährung bei Krebspatienten

\title{
Frühzeitig reagieren
}

Die Mangelernährung von Tumorpatienten wird nach wie vor unterschätzt. Dabei gilt es, möglichst früh gegenzusteuern. Eine Schlüsselrolle übernehmen hier onkologische Pflegekräfte.

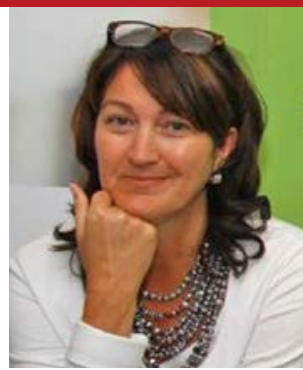

Kerstin Paradies Vorstandssprecherin der KOK

? Frau Paradies, wie wichtig ist das Thema Mangelernährungfür Pflegende? Paradies: Die Ernährung steht leider bisher nicht im Fokus der Therapieplanung. Jeder Onkologe versucht, die optimale Therapie für den Tumorpatienten zu finden. Aber nicht nur die Gabe von Zytostatika ist wichtig für die Gesundung, sondern auch das, was sonst mit dem Menschen passiert. Doch Pflegekräfte sind dafür ausgebildet, den Patienten ganzheitlich zu sehen. Inzwischen gibt es zwar immer mehr Ärzte, die sich der Ernährung annehmen. Aber: Wir sind die erste Anlaufstelle! Wir merken nicht erst, wenn der Patient mangelernährt ist, sondern schon deutlich früher. Wenn der Arzt den Patienten fragt, wie es ihm geht - alles super. Dann kommen sie zu uns und berichten von Übelkeit, Erbrechen, Durchfall, Appetitlosigkeit.

? Werden die Folgen verkannt?

Paradies: Für viele Patienten gehören Probleme wie Übelkeit, Erbrechen und Gewichtsverlust zu einer Chemotherapie einfach dazu, schließlich erhalten sie ja ein Zellgift. Und unsere Patientinnen - ich arbeite gynäkologisch-onkologisch - finden es oft ganz okay, unter der Chemotherapie mal etwas abzunehmen. Diesen Frauen müssen wir klarmachen, dass sie wichtige Muskelmasse verlieren.

? Woran erkennen Sie, dass ein Patient mangelernährt ist?

Paradies: Das merkt man schon sehr früh. In unserer Praxis werden die Patientinnen bei jedem Kontrollbesuch gewogen und das Ergebnis dokumentiert. Außerdem haben wir alle 14 Tage eine Ernährungsberaterin in der Praxis, die auch Bioimpedanz-Messungen durchführt.
? Was können Betroffene tun, damit Ernährungsprobleme frühzeitig erkannt werden?

Paradies: Sich mit uns zusammensetzen, darüber sprechen. Wie waren die Essgewohnheiten vor der Erkrankung, wie sind sie jetzt? Gibt es Nebenwirkungen der Chemotherapie, die sich ungünstig auswirken, z.B. eine Mukositis? Wie ist die Situation zu Hause mit Einkaufen und Kochen? Dann können wir ihn beraten und die vorhandenen Probleme lösen.

Als hilfreich erweisen sich auch einfache Ernährungstipps: kleine Mahlzeiten über den Tag zu verteilen, zusätzlich ein Stück Butter ans Gemüse zu geben, bei Appetit, mal ein Stück Kuchen zu essen. Bei größerem Gewichtsverlust schalten wir immer einen Ernährungsberater ein. Und wir weisen unsere ärztlichen Kollegen darauf hin, wenn sich in punkto Mangelernährung etwas zusammenbraut.

? Gibt es große Hindernisse zu überwinden, wenn eine parenterale Ernährungstherapie nötig wird?

Paradies: Im stationären Bereich eher nicht, weil da Ernährungsberater im Haus sind. Im ambulanten Bereich müssen wir manchmal Überzeugungsarbeit leisten, wenn etwas getan werden muss. Gerade hier kommt es darauf an, entsprechende Netzwerke mit Ernährungsberatern und Pflegediensten aufzubauen. Denn der Pflegedienst, der abends zum Patienten kommt und den Ernährungsbeutel anhängt, muss Erfahrung im Anstechen eines Ports haben. Auch gute Kontakte zu Firmen wie Baxter sind da sehr hilfreich.

Nach Informationen der Baxter Deutschland GmbH 